\title{
Detection of Tumor in Liver Using Image Segmentation and Registration Technique
}

\author{
Priyanka Kumar ${ }^{1}$, Shailesh Bhalerao ${ }^{2}$ \\ ${ }^{1}$ Department of Electronics Engineering, G.H. Raisoni College of Engineering, Nagpur, India \\ ${ }^{2}$ Department of Electronics Engineering, G.H. Raisoni College of Engineering, Nagpur, India,
}

\begin{abstract}
Now-a-days, there is rise in the death rate of patients suffering from liver cancer. The liver cancer rate is increasing year by year. Generally, liver cancer's death rate is very high because the disease causes no symptoms, so it's often not caught until it's in final stages. The Canadian Cancer Society says, if we catch someone's liver cancer early, their chance of defeating the disease is 70 to 80 per cent. If the disease is caught late, the average person survives about a year after diagnosis. We propose an algorithm for liver cancer detection which is based on concepts of fuzzy logic and neural network. Neuro-fuzzy (NF) systems are suitable tools to deal with uncertainty found in the process of extracting useful information from images. In this work, the liver tumor is detected through the medical images in three phases, pre-processing phase, processing phase and detection phase. Initially in the pre-processing phase, a set of medical images is filtered for removing noise. Then the filtered image is segmented automatically using fuzzy logic, neural network and windowing technique. In the detection phase neuro-fuzzified segmented images of CT and MRI is registered to obtain the tumor. The result is obtained for few different set of database.
\end{abstract}

Keywords: Image segmentation, Image registration, K-means clustering, Neural Network, Fuzzy logic

\section{Introduction}

Automatic diagnostic systems are an important application of analysis of database and pattern recognition, which aims at assisting physicians in making diagnostic decisions [1]. Automated diagnosis is especially used to detect the various types of cancers. Because of the fact that, cancer is the second leading cause for death of both men and women in the world, and is expected to become the first cause of death in the next few decades [2]. Automated diagnostic systems have been applied to and are of interest for a variety of medical data,[1]including medical signals and medical images. In medical science if doctors find any abnormal behavior in human being, they ask for certain diagnosis tests to be done, prior to confirmation of any disease. There are various kinds of tests stipulated for different organs. Since, we are going to deal with Liver cancer, we are concerned about the liver cancer diagnosis tests. There are various tests for diagnosis of liver cancer. Among these tests CT scan and MRI is of utmost important. Generally, every doctor ask for CT scan to look for the tumor in the liver. If they find the liver affected, then later on they ask for MRI to get detail knowledge about the tumor in the liver. Because MRI provides a better view and proper tumor location.

For all the steps mentioned above doctors go for manual technique. They look into the images manually and tell about the affected liver, the liver tumor and there location. For all this, a lot of time is consumed. There are also some cases where doctors do not mention all these detail to the patient. So we have made an approach to design an algorithm where liver tumor is detected automatically. Both the CT and MRI image is used for registration and detect the tumor automatically.

\section{Image Segmentation}

Image segmentation is an important process to extract information from complex medical images. Segmentation has wide application in medical field. The main objective of image segmentation is to partition an image into mutually exclusive and exhausted regions such that each region of interest is spatially contiguous and the pixels within the region are homogeneous with respect to a predefined criterion. Widely used homogeneity criteria include values of intensity, texture, color, range, surface normal and surface curvatures. Several diagnostics are based on proper segmentation of the digitized image. Segmentation of medical images is needed for applications involving estimation of the boundary of an object, classification of tissue abnormalities, shape analysis, contour detection. Many approaches are based on fuzzy logic, K means and Neural Networks $(\mathrm{NN})$, etc [3]. Image segmentation and its performance evaluation are important fields in image processing and, because of the complexity of the medical images, segmentation of medical image is still a challenging problem[13]. 


\subsection{SEGMENTATION BASED ON CLUSTERING}

Clustering is an unsupervised learning task, where one needs to identify a finite set of categories known as clusters to classify pixels [4].Clustering use no training stages rather train themselves using available data. Clustering is mainly used when classes are known prior. A similarity based criteria is defined between pixels [2], and then similar pixels are brought together in groups to form clusters. The grouping of pixels into clusters is based on the principle of maximizing both the intra class similarity and the inter class similarity. The quality of a clustering result depends on both the similarity measure used by the method and its implementation. Clustering algorithms are classified as hard clustering, k- means clustering, fuzzy clustering, etc [4].

\subsection{K-MEANS CLUSTERING}

$k$-means clustering is a method of vector quantization, originally from signal processing, that is popular for cluster analysis in data mining. $k$-means clustering aims to partition $n$ observations into $k$ clusters in which each observation belongs to the cluster with the nearest mean, serving as a prototype of the cluster. This results in a partitioning of the data space into Voronoi cells. Given a set of observations $(\mathrm{x} 1, \mathrm{x} 2, \ldots, \mathrm{x} n)$, where each observation is a $d$-dimensional real vector, $k$-means clustering aims to partition the $n$ observations into $k$ sets $(k$ $\leq n) \mathrm{S}=\{S 1, S 2, \ldots, S k\}$ so as to minimize the with in cluster sum of squares (WCSS):

$$
\underset{\mathbf{S}}{\arg \min } \sum_{i=1}^{k} \sum_{\mathbf{x}_{j} \in S_{i}}\left\|\mathbf{x}_{j}-\boldsymbol{\mu}_{i}\right\|^{2}
$$

where $\mu i$ is the mean of points in $\mathrm{Si}$ [4].

$\mathrm{K}$-means is a type of exclusive clustering algorithms [5, 6]. $\mathrm{K}$ means algorithm starts clustering by assigning $\mathrm{k}$ initial central points, either randomly or using heuristic data. It then groups each image pixel under the central point which it is closest to. Next, it calculates new central points by taking average of the pixels grouped under each central point. The two former algorithmic steps are repeated alternately until convergence. The limitations of K-means clustering is that number of iterative rounds may be required.

\section{Image Registration}

Finding the differences between two similar or identical images can be noticed by eye, but sometimes those differences are embedded in the image and takes a lot of time to be found, using some developed MATLAB code may make such operation rapid and accurate[7]. Medical image registration between different modalities or different images is very effective for comprehensive and precious diagnosis and treatment [8-10]. Image registration is the process of overlaying two or more images of the same scene taken at different times, from different viewpoints, and/or by different sensors. It geometrically aligns two images - the reference and sensed images. The present differences between images are introduced due to different imaging conditions. Image registration is a crucial step in all image analysis tasks in which the final information is gained from the combination of various data sources like in image fusion, change detection, and multichannel image restoration. Typically, registration is required in remote sensing (multispectral classification, environmental monitoring, change detection, image mosaicing, weather forecasting, creating super-resolution images, integrating information into geographic information systems (GIS)), in medicine (combining computer tomography (CT) and NMR data to obtain more complete information about the patient, monitoring tumor growth, treatment verification, comparison of the patient's data with anatomical atlases), in cartography (map updating), and in computer vision (target localization, automatic quality control), to name a few[11]. Image registration is required in medical applications, it depends on combining computer tomography (CT) and NMR data to obtain more complete information about the patient[9]

The proposed algorithm is as given below:

\section{Proposed Method}

\subsection{Preprocessing stage:}

Generally medical images like MRI, CT etc. always contain a significant amount of noise caused by operator performance, equipment, and the environment, which can lead to serious inaccuracies. So filtration is required to remove this noise, so that more accurate values are reached.

Step 1: The given image is read. After reading the image, it is converted into matrix form. Each pixel in the matrix is in the range $0-255$. 


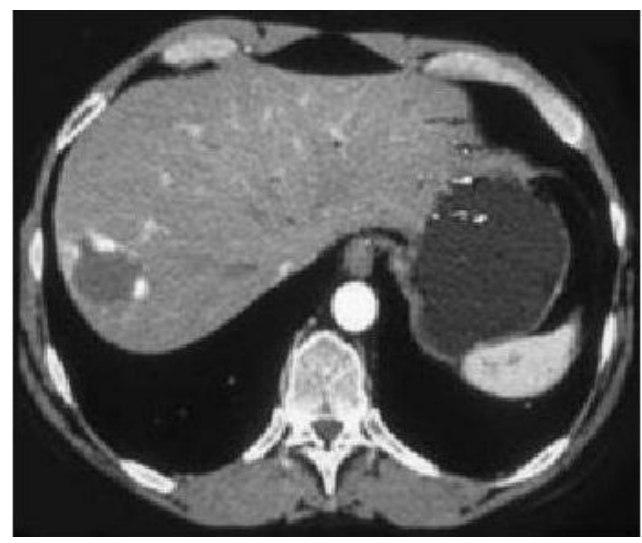

Figure1. Input image

Step 2: The input image is then median filtered to remove noise.

DENOISING:

Most of the images are affected by noise and artifacts caused by the various acquisition techniques and, hence, an effective technique for removing noise is necessary for medical images, particularly in computed tomography, which is a significant and most general modality in medical imaging. Hence, denoised and quality of images are enhanced [7].

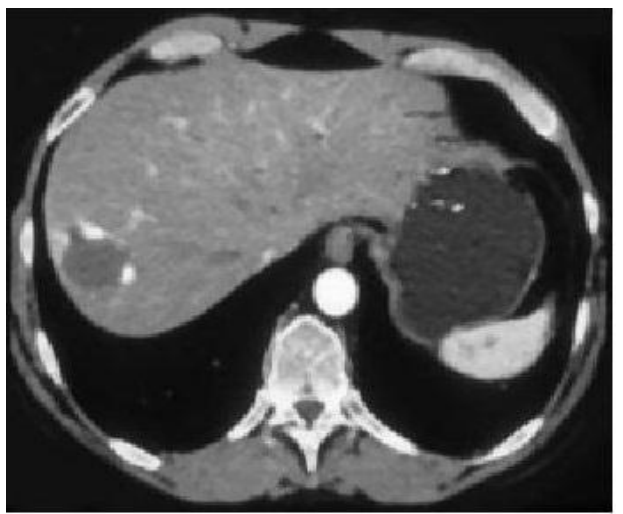

Figure2. Filtered image

\subsection{Processing stage}

Step 3: Then the membership functions are defined for the filtered image and clusters are formed using fuzziness The fuzzy interference system is created.

Step 4: The number and type of Membership functions for the input image is decided by tuning the membership functions. Fuzzy rules are also required for it.

For clustering purpose the following IF-THEN fuzzy rules are used in the algorithm:

\begin{tabular}{|l|l|l|}
\hline Mean value & Standard deviation & Edge pixel \\
\hline Low & Low & Not an edge pixel \\
\hline Medium & Low & An edge pixel \\
\hline High & Low & Not an edge pixel \\
\hline Low & High & Not an edge pixel \\
\hline Medium & High & Not an edge pixel \\
\hline High & High & Not an edge pixel \\
\hline
\end{tabular}

Table I: IF-THEN rules

The given rules are plotted along the axes: 


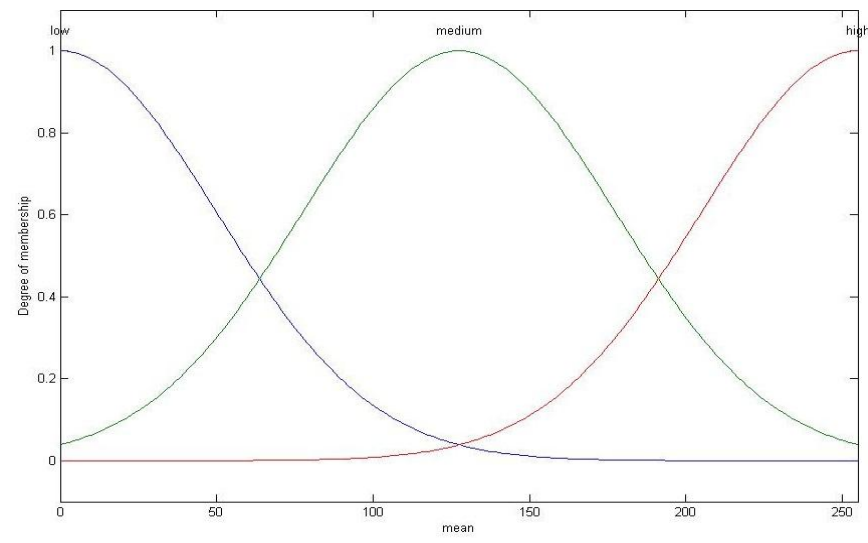

Figure3: Degree of membership vs mean

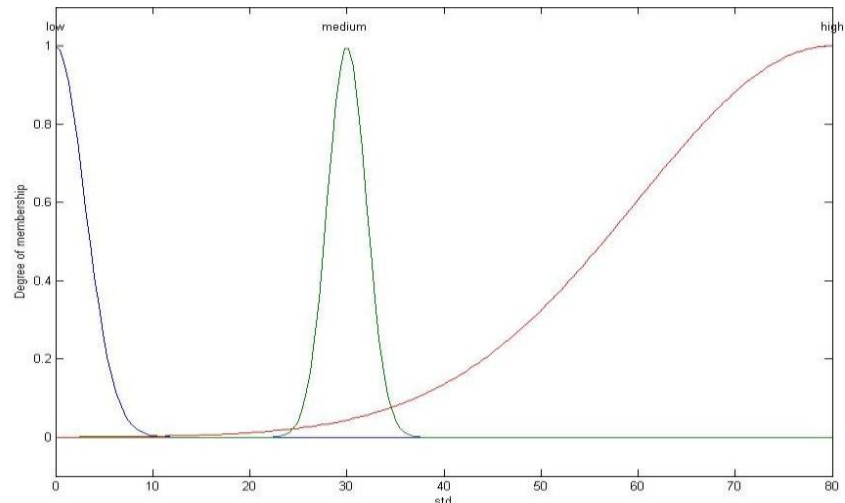

Figure4: Degree of membership vs standard deviation

Step 5: Based on the above rules developed Fuzzy classification is applied on the corresponding pixel values of the input image which gives a fuzzy set represented by a membership function. The rules are checked using rule viewer and surface viewer.

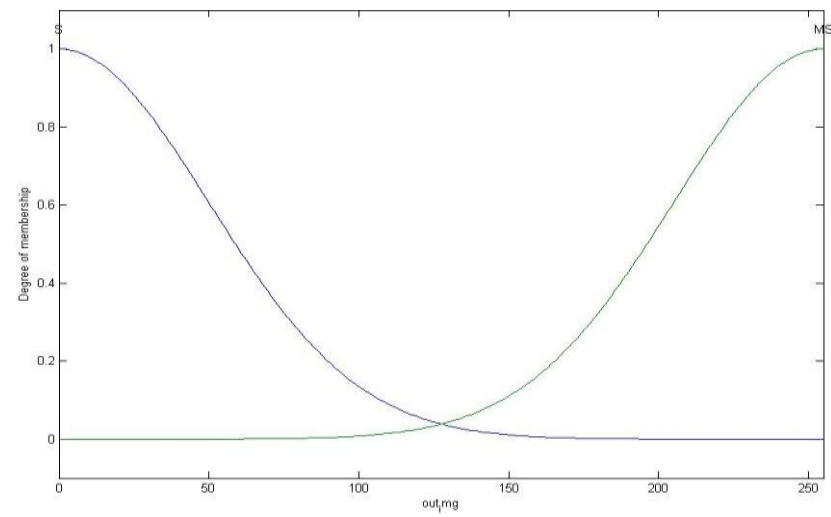

Figure5: Degree of membership vs pixels of output image

Step 6: Cluster centers are chosen and the Features are extracted. Neighborhood attraction is considered to exist between neighboring pixels. This is considered as a feature for extraction.

Step 7: Based on similarity measures, clustering is done. During clustering, each pixel attempts to attract its neighboring pixels toward its own cluster. This neighborhood attraction depends on two factors; the pixel intensities or feature attraction, and the spatial position of the neighbors or distance attraction, which also depends on the neighborhood structure.

Step 8: Membership function and cluster centers are updated in according.

Step 9: Cost function is calculated and weights are updated.

Step 10: Stopping condition is tested and the column form is converted to matrix form and display the segmented image. 

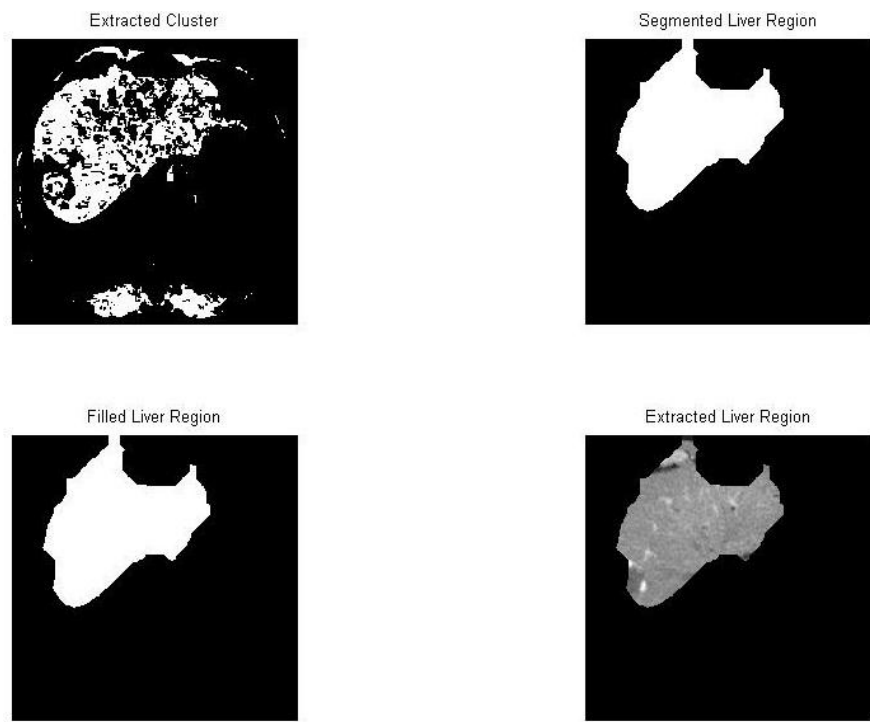

Figure6.Semented image

\subsection{Detection phase}

Step 11: Detection of probable tumor region without registration.

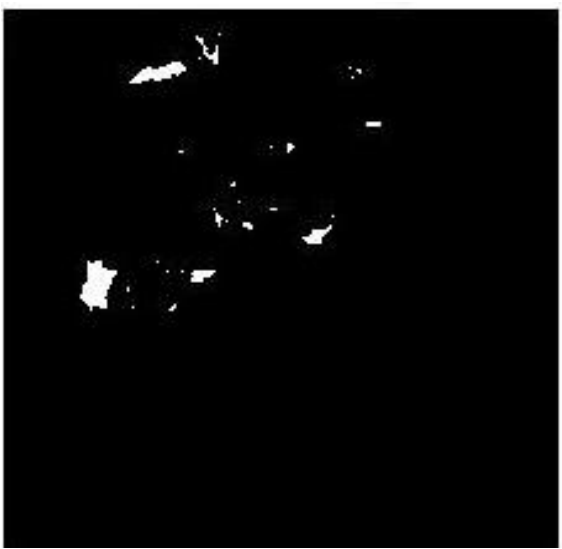

Figure6. Probable tumor region for CT image 1

Step 12: Tumor detection using registration technique: Registration of the CT and MRI image is done in GUI to obtain the tumor. Above mentioned steps are followed in GUI too. Final results are shown in the result section shown below.

\section{Results}

Registration has been done for different set of data. Each set consists of an MRI image and a CT image. For different set of images available, registration has been done. Tumor for each respective set has been detected using the algorithm described above. The results for different dataset have been shown below.

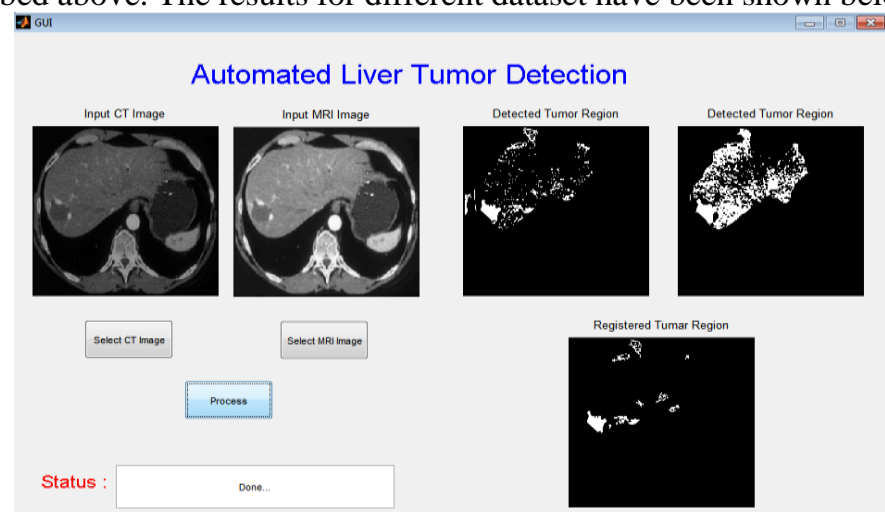

Figure7. Result for data set 1 


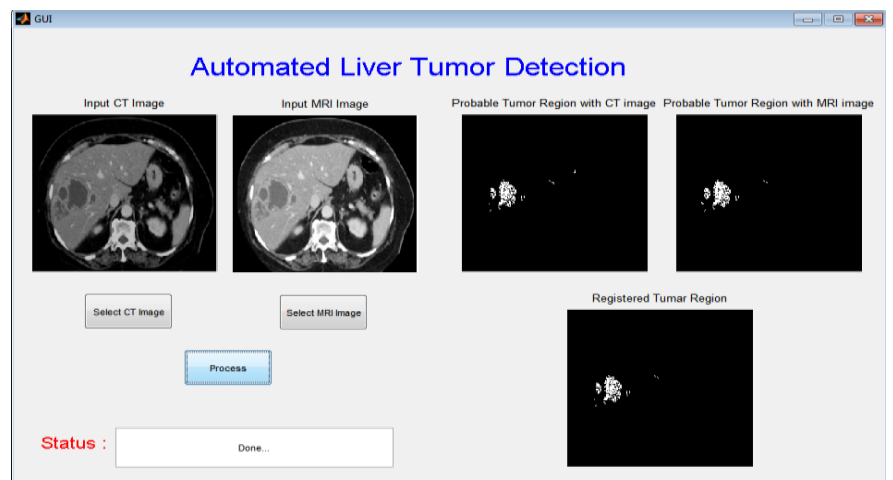

Figure8. Result for data set 2

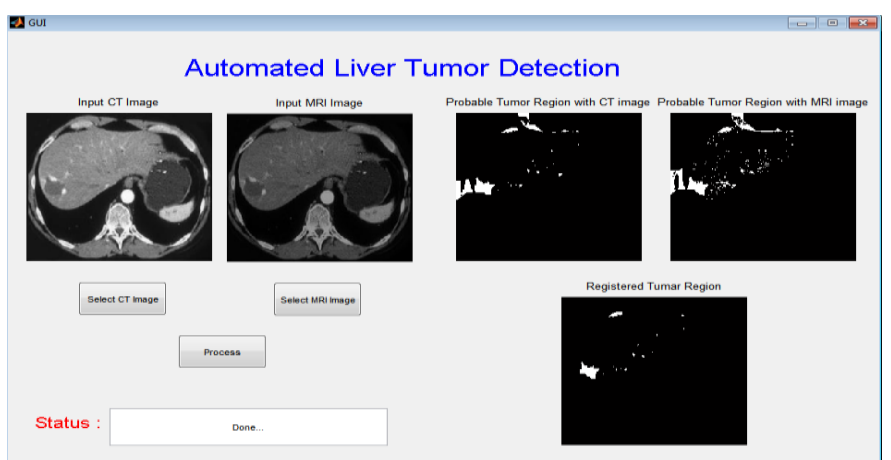

Figure9. Result for data set 3

\section{Conclusion And Discussion}

In medical science since doctors go for a manual technique to detect and locate the liver tumor, here is an approach to detect the tumor automatically. Tumor detection using both CT and MRI image has been done. The system has been implemented in the working platform MATLAB R2012b and the publicly available data are utilized.

Future work will focus on applying this algorithm for all the type of cancers which is diagnosed using medical image. An approach will also be made to design a particular system for this.

\section{References}

[1]. Übeyli ED, Adaptive neuro-fuzzy inference systems for automatic detection of breast cancer, J Med Syst2009;33:353 [PubMed]

[2]. Abarghouei AA, Ghanizadeh A, Sinaie S, Shamsuddin SM, A Survey of Pattern Recognition Applications in Cancer Diagnosis, in International Conference of Soft Computing and Pattern Recognition. IEEE Conferences. 2009:448-53.

[3]. C. Venkatesh, Fahimuddin Shaik, Ghouse Mohammed Imran, T.Haneesh, fuzzy-neuro logic in segmentation of mri images, IEEEInternational Conference On Advances In Engineering, Science And Management (ICAESM -2012) March 30, 31, 2012

[4]. V. K. Dehariya, S. K. Shrivastava, R. C. Jain, Clustering of Image Data Set Using K-Means and Fuzzy KMeans Algorithms, International conference on CICN, pp. 386- 391, 2010.

[5]. S.Saheb Baha and Dr. K.Satya Prasad, Automatic detection of Hard Exudates in Diabetic Retinopathy using Morphological segmentation and Fuzzy Logic in [JCSNS International Journal of Computer Science and Network Security, VOL.8 No.12, December 2008.

[6]. Juraj Horvath, Image Segmentation using Fuzzy C-means,SAMI 2006.

[7]. Hazem (Moh'd Said) Hatamleh, Image Processing of Two Identical and Similar Photos, Journal of Information Engineering and Applications ISSN 2224-5782 (print) ISSN 2225-0506 (online) Vol.3, No.1, 2013

[8]. R. P. Woods, J. C. Mazziotta, S. R. Cherry, MRI-PET registration with automated algorithm, J. Comput. Assist. Tomogr. Vol. 17, pp. 536-546 1993

[9]. B. A. Adrekani, M Braun, B. F. Hutton, I Kanno, and H. Iida, "A fully automatic multimodality image registration algorithm," J. Comput. Assist. Tomogr. Vol. 19, pp. 615-623 1995

[10]. K. S. Friston, J. Ashburner, C. D. Frith, J.-B. Poline, J. D. Heather, and R. S. J. Frackowiak, "Spatial registration and normalization of images," Human Brain Mapping, vol. 2 pp. 165-189 (1995)

[11]. Barbara Zitova, Jan Flusser, Image registration methods: a survey, Image and Vision Computing 21 (2003) 977-1000

[12]. Yen-Wei Chen, Katsumi Tsubokawa, Amir H. Foruzan, Shigehiro Morikaw, and Yoshimasa Kurumi, Image segmentation and registration techniques for mr-guided liver cancer surgery, 978-1-4673-2349-9/12/\$31.00 @2012 IEEE

[13]. Zhang, Y. J, An Overview of Image and Video Segmentation in the last 40 years, Proceedings of the $6^{\text {th }}$ International Symposium on Signal Processing and Its Applications, pp. 144-151, 2001.

[14]. Monireh Sheikh Hosseini and Maryam Zekri, Review of Medical Image Classification using the Adaptive Neuro-Fuzzy Inference System, J Med Signals Sens. 2012 Jan-Apr; 2(1): 49-60. 